\title{
EQUALLY DISTRIBUTED CORRESPONDENCES
}

\author{
Sergiu HART \\ Department of Mathematics, Tel Aviv University, Tel Aviv, Israel \\ Elon KOHLBERG \\ Department of Economics, Harvard University, Cambridge, Mass., U.S.A. \\ Received August 1973, revised version received February 1974 \\ The distribution $\mathscr{D} \varphi$ of a correspondence $\varphi$ is defined, and its connection with the set $\mathscr{D} \mathscr{L}_{\varphi}$ of \\ distributions of its integrable selections is explored. The main result is that if $\varphi_{1}$ and $\varphi_{2}$ are \\ equally distributed, i.e., if $\mathscr{D} \varphi_{1}=\mathscr{D} \varphi_{2}$, then $\mathscr{D} \mathscr{L}_{\varphi_{1}}$ and $\mathscr{D} \mathscr{L}_{\varphi_{2}}$ have the same closure in the
} weak convergence topology.

\section{Introduction}

The study of measurable and integrable selections from a correspondence (set-valued function) has been of interest for some time [e.g., see Aumann (1965), Hildenbrand (1974), etc.].

Here we take up the study of distributions of such selections. The motivation for doing this comes from Mathematical Economics [see Kannai (1970, section 7); the reader interested in the economic applications is referred to Hart et al. (1974)].

The first question we consider is the following: Does the distribution of a correspondence determine the distributions of its selections? If the underlying space contains atoms, then of course we cannot expect an affirmative answer (e.g., consider the correspondence whose image is always the set $\{1,2\}$, once on a space consisting of one atom, and once on a space consisting of two atoms). But what if the underlying space is atomless?

Consider the following example [which is essentially a reformulation of an example due to G. Debreu - see Kannai (1970, section 7)].

Let

$$
\varphi_{1}(t)=\{t,-t\} \text { for all } t \in[0,1]
$$

*This research was carried out while both authors were participating at the Workshop on Mathematical Economics at the Institute for Mathematical Economics, University of Bielefeld, Rheda, W. Germany, June-July 1973. The research of the first author is supported by the National Council for Research and Development in Israel. 
and

$$
\varphi_{2}(t)= \begin{cases}\{2 t,-2 t\}, & \text { for } t \in\left[0, \frac{1}{2}\right], \\ \{2 t-1,1-2 t\}, & \text { for } t \in\left(\frac{1}{2}, 1\right] .\end{cases}
$$

Clearly, $\varphi_{1}$ and $\varphi_{2}$ must be equally distributed by any reasonable definition of this concept. However, the distributions of their measurable selections are not the same: let

$$
f(t)=\left\{\begin{aligned}
2 t, & \text { for } t \in\left[0, \frac{1}{2}\right], \\
1-2 t, & \text { for } t \in\left(\frac{1}{2}, 1\right],
\end{aligned}\right.
$$

then $f$ is a measurable selection from $\varphi_{2}$, but there is no measurable selection from $\varphi_{1}$ with the same distribution.

The above example, in giving a negative answer to our question, raises serious difficulties as to possible applications of the concept of 'distributions of selections'. However, most of the difficulties can be eliminated provided the following is true: if $\varphi_{1}$ and $\varphi_{2}$ are equally distributed correspondences, then the closures of the distributions of their selections are equal. Theorem 1 is the precise formulation of this statement.

In Theorem 3 we show that when the measurable selections are restricted to have a constant integral, the closure of their distributions still depends only on the distribution of the correspondence [this restriction is of importance in Mathematical Economics; indeed, our Theorem 3 is a well-known conjecture of R.J. Aumann - see Kannai (1970, section 7)].

\section{Preliminaries}

We denote by $\mathbf{R}^{l}$ the $l$-dimensional Euclidian space, and by $\mathscr{B}\left(\mathbf{R}^{l}\right)$ the $\sigma$ algebra of its Borel subsets.

Let $(A, \mathscr{A}, v)$ be a measure space; in the following, all measure spaces will be assumed to be complete [i.e., $\mathscr{A}$ contains all subsets of null sets - see Hildenbrand (1974, D.I)]. Null sets will be systematically ignored.

Let $f:(A, \mathscr{A}, v) \rightarrow \mathbf{R}^{l}$ be a measurable function. The distribution $\mathscr{D} f$ of $f$ is defined as $v \circ f^{-1}$ [i.e., the induced measure on $\mathscr{B}\left(\mathbf{R}^{l}\right)$ ]. The sequence ${ }^{1}\left\{f_{n}\right\}$ converges to $f$ in distribution if the sequence $\left\{\mathscr{D} f_{n}\right\}$ converges weakly to $\mathscr{D} f$, where weak convergence of measures is defined as usual by $\mu_{n} \stackrel{W}{\rightarrow} \mu$ if $\int h \mathrm{~d} \mu_{n} \rightarrow$ $\int h \mathrm{~d} \mu$ for all real, continuous and bounded functions $h$. The topology of weak convergence can be metrized, e.g., by the Prohorov metric $\rho$, defined as

$$
\begin{gathered}
\rho\left(\mu_{1}, \mu_{2}\right)=\inf \left\{\varepsilon>0 \mid \mu_{1}(B) \leqq \mu_{2}\left(B_{\varepsilon}\right)+\varepsilon \text { and } \mu_{2}(B) \leqq \mu_{1}\left(B_{\varepsilon}\right)+\varepsilon\right. \\
\text { for all Borel subsets } B\},
\end{gathered}
$$

where

$$
\boldsymbol{B}_{\varepsilon}=\{x \mid \mathrm{d}(x, B)<\varepsilon\} .
$$

\footnotetext{
${ }^{1}$ Note that the $f_{n}$ 's can be defined on different spaces, but their range must always be in the same space.
} 
Let $^{2} \varphi:(A, \mathscr{A}, v) \rightarrow \mathbf{R}^{l}$ be a correspondence, i.e., $\varphi(a)$ is a non-empty subset of $\mathbf{R}^{l}$ for all $a \in A$. The graph of $\varphi$ is the set

$$
G_{\varphi}=\{(a, x) \mid x \in \varphi(a)\} .
$$

$\varphi$ has measurable graph [or 'is Borel-measurable', as in Aumann (1965)] if $G_{\varphi} \in \mathscr{A} \otimes \mathscr{B}\left(\mathbf{R}^{l}\right) . \varphi$ is integrably bounded if there exists an integrable function $h:(A, \mathscr{A}, v) \rightarrow \mathbf{R}^{l}$ such that $-h(a) \leqq x \leqq h(a)$ for all $x \in \varphi(a)$ and for all $a \in A . \varphi$ is closed-valued if $\varphi(a)$ is a closed set (in $\mathbf{R}^{l}$ ) for every $a \in A$.

The set of all integrable selections from $\varphi$ [i.e., all integrable functions $f$ such that $f(a) \in \varphi(a)$ for all $a \in A]$ is denoted $\mathscr{L}_{\varphi}$, and $\int \varphi=\left\{\int f \mid f \in \mathscr{L}_{\varphi}\right\}$. For every $B$, let

$$
\varphi^{-1}(B)=\{a \in A \mid \varphi(a) \cap B \neq \phi\}
$$

[the (weak) inverse of $\varphi$ ].

All the definitions up to now are standard [see, e.g., Billingsley (1968) and Hildenbrand (1974)]. At this point we must make precise the notion of 'equally distributed correspondences'. We therefore define the distribution $\mathscr{D} \varphi$ of the correspondence $\varphi$ having a measurable graph, by $\mathscr{D} \varphi=\nu \circ \varphi^{-1}$. This definition is meaningful since by the projection theorem [Hildenbrand (1974, D.I(11))], if $\varphi$ has a measurable graph then $\varphi^{-1}(B)$ is measurable for all $B \in \mathscr{B}\left(\mathbf{R}^{l}\right)$. Note that $\mathscr{T} \varphi$ is not necessarily additive; if, for all $a \in A, \varphi(a)$ consists of just one point, then this definition coincides with the usual one for functions. We say that $\varphi_{1}$ and $\varphi_{2}$ are equally distributed if $\mathscr{D} \varphi_{1}=\mathscr{D} \varphi_{2}$.

As a Corollary to Theorem 4, we shall prove that, at least for closed-valued correspondences, our definition of equally distributed correspondences coincides with the usual one of functions with the same distribution, when regarding the correspondences as point-valued functions into the set of (closed) subsets of $\mathbf{R}^{l}$.

The following notations will be used: | for set-theoretic substraction, $K^{\mathrm{c}}$ for the complement of $K$, and $\int f$ for $\int_{A} f(a) \mathrm{d} v(a)$. For a set $\mathscr{F}$ of functions with the same range, $\mathscr{D} \mathscr{F}$ will denote the set of their distributions, i.e., $\mathscr{D} \mathscr{F}=\{\mathscr{D} f \mid f \in$ $\overline{\mathscr{F}}\}$, and $\overline{\mathscr{D}} \mathscr{F}$ will be its closure with respect to the weak convergence of measures.

\section{Statement of the results}

In Theorems $1-3,\left(A_{i}, \mathscr{A}_{i}, v_{i}\right)$ (for $\left.i=1,2\right)$ will be non-atomic complete probability measure spaces, and $\varphi_{i}:\left(A_{i}, \mathscr{A}_{i}, v_{i}\right) \rightarrow \mathbf{R}^{l}$ correspondences with measurable graphs.

Theorem 1. Let $\varphi_{1}$ and $\varphi_{2}$ be integrably bounded. If $\varphi_{1}$ and $\varphi_{2}$ are equally distributed, then the sets $\mathscr{D} \mathscr{L}_{\varphi_{1}}$ and $\mathscr{D} \mathscr{L}_{\varphi_{2}}$ of distributions of the integrable

\footnotetext{
${ }^{2}$ We write $\varphi: A \rightarrow \mathbf{R}^{l}$ also for correspondences, but then we mean $\varphi(a) \subset \mathbf{R}^{l}$ for all $a \in A$; no confusion will arise, since correspondences are always denoted by the Greek letters $\varphi$ or $\psi$.
} 
selections of $\varphi_{1}$ and $\varphi_{2}$, respectively, have the same closure with respect to the weak convergence, i.e.,

$$
\overline{\mathscr{Q} \mathscr{L}_{\varphi_{1}}}=\overline{\mathscr{D} \mathscr{L}_{\varphi_{2}}} .
$$

The following theorem is an immediate consequence of Theorem 1 .

Theorem 2. Let $\varphi_{1}$ and $\varphi_{2}$ be integrably bounded and closed-valued. If $\varphi_{1}$ and $\varphi_{2}$ are equally distributed, then $\int \varphi_{1}=\int \varphi_{2}$.

Theorem 3. Let $\varphi_{1}$ and $\varphi_{2}$ be integrably bounded and closed-valued, and let $x \in \mathbf{R}^{l}$. If $\varphi_{1}$ and $\varphi_{2}$ are equally distributed, then the sets of distributions of the integrable selections of $\varphi_{1}$ and $\varphi_{2}$ with integral equal to $x$, have the same closure with respect to the weak convergence, i.e.,

$$
\overline{\mathscr{D}\left\{f \in \mathscr{L}_{\varphi_{1}} \mid \int f=x\right\}}=\overline{\mathscr{D}\left\{g \in \mathscr{L}_{\varphi_{2}} \mid \int g=x\right\}} .
$$

Clearly, if $\varphi_{i}=\psi \circ h_{i}$, where the functions $h_{1}$ and $h_{2}$ have the same distribution, then $\varphi_{1}$ and $\varphi_{2}$ are equally distributed (this is, indeed, the case in Mathematical Economics). The following decomposition theorem asserts that this is the general structure of equally distributed correspondences.

Theorem 4. For $i=1,2$, let $\left(A_{i}, \mathscr{A}_{i}, v_{i}\right)$ be complete probability measure spaces, and let $\varphi_{i}:\left(A_{i}, \mathscr{A}_{i}, v_{i}\right) \rightarrow \mathbf{R}^{l}$ be closed-valued correspondences with measurable graphs. Then $\mathscr{D} \varphi_{1}=\mathscr{D} \varphi_{2}$ if and only if there exist a measurable space $(E, \mathscr{E})$, a correspondence $\psi:(E, \mathscr{E}) \rightarrow \mathbf{R}^{l}$ with measurable graph, and measurable functions $h_{i}:\left(A_{i}, \mathscr{A}_{i}, v_{i}\right) \rightarrow(E, \mathscr{E})$ such that $\varphi_{i}=\psi \circ h_{i}$ for $i=1,2$, and $\mathscr{D} h_{1}=\mathscr{D} h_{2}$.

Let $\mathscr{C}^{l}$ denote the family of all closed and non-empty subsets of $\mathbf{R}^{l}$, endowed with the topology of 'closed convergence' [e.g., see Hildenbrand (1974, B.II)], and let $\mathscr{B}\left(\mathscr{C}^{l}\right)$ be its Borel $\sigma$-field (i.e., the $\sigma$-field generated by the open subsets of $\mathscr{C}^{l}$ ); a discussion of these concepts will be found before the proof of Theorem 4.

Corollary. For $i=1,2$, let $\left(A_{i}, \mathscr{A}_{i}, v_{i}\right)$ be complete probability measure spaces, and let $\varphi_{i}:\left(A_{i}, \mathscr{A}_{i}, v_{i}\right) \rightarrow \mathbf{R}^{l}$ be closed-valued correspondences with measurable graphs. Let $h_{i}:\left(A_{i}, \mathscr{A}_{i}, v_{i}\right) \rightarrow\left(\mathscr{C}^{l}, \mathscr{B}\left(\mathscr{C}^{l}\right)\right)$ be the corresponding point-valued functions, i.e., the functions defined by $h_{i}(a)=\varphi_{i}(a) \in \mathscr{C}^{l}$ for all $a \in A_{i}$. Then $\mathscr{D} \varphi_{1}=\mathscr{D} \varphi_{2}$ if and only if $\mathscr{D} h_{1}=\mathscr{D} h_{2}$.

\section{Proof of the results}

In the proof of Theorem 1 we rely on the following lemma: 
Lemma. Let $(A, \mathscr{A}, v)$ be a non-atomic measure space. Let $\left\{S_{i}\right\}_{i=0}^{m} \subset \mathscr{A}$ and $\left\{\alpha_{i}\right\}_{i=0}^{m}, \alpha_{i} \geqq 0$ be such that

$$
v\left(\bigcup_{i \in I} S_{i}\right) \geqq \sum_{i \in I} \alpha_{i}, \text { for all } I \subset\{0,1 \ldots, m\},
$$

and

$$
\nu\left(\bigcup_{i=0}^{m} S_{i}\right)=\sum_{i=0}^{m} \alpha_{i}
$$

Then there exist disjoint sets $\left\{T_{i}\right\}_{i=0}^{m}$ such that $T_{i} \subset S_{i}$ and $v\left(T_{i}\right)=\alpha_{i}$ for all $i=0,1, \ldots, m$.

The proof of this lemma may be carried out in complete analogy with the proof of a well known result in Combinatorics [see Halmos and Vaughan (1950)]. Since it is quite short, we repeat it here.

Proof. We use induction on $m$. For $m=0$ it is trivial. Let $m>0$. We distinguish two cases.

Case (i). For some $I \notin\{0,1, \ldots, m\}$, there is equality in (1). Then it is easily verified that both $\left\{S_{i}, \alpha_{i}\right\}_{i \in I}$ and $\left\{S_{j} \backslash \bigcup_{i \in I} S_{i}, \alpha_{j}\right\}_{j \notin I}$ satisfy (1) and (2), and we may apply the induction hypothesis separately to each one of them.

Case (ii). For all $I \ddagger\{0,1, \ldots, m\}$, there is strict inequality in (1). In particular, $v\left(S_{0}\right)>\alpha_{0}$ and $v\left(S_{0} \mid \bigcup_{i \neq 0} S_{i}\right)<\alpha_{0}$. Since $v$ is non-atomic, we may find an $S_{0}^{\prime}$ such that

$$
S_{0} \mid \bigcup_{i \neq 0} S_{i} \subset S_{0}^{\prime} \subset S_{0},
$$

and the replacement of $S_{0}$ by $S_{0}^{\prime}$ will preserve all inequalities in (1), but at least one of them will be an equality. Clearly, (2) is still valid, and we may proceed as in case (i). Q.E.D.

\section{Proof of Theorem 1}

Let $f \in \mathscr{L}_{\varphi_{1}}$ and let $\varepsilon>0$. We must find $g \in \mathscr{L}_{\varphi_{2}}$ such that $\rho(\mathscr{D} f, \mathscr{D} g)<\varepsilon$.

Since $f$ is integrable, there is a compact set $K$ in $\mathbf{R}^{l}$ such that $v_{1}\left(f^{-1}\left(K^{\circ}\right)\right)<\varepsilon$. Let $K_{0}=K^{\mathrm{c}}$ and let $K_{1}, K_{2}, \ldots, K_{m}$ be a partition of $K$ into disjoint Borel sets of diameter less than $\varepsilon$.

Define $\alpha_{i}=v_{1}\left(f^{-1}\left(K_{i}\right)\right)$ and $S_{i}=\varphi_{2}^{-1}\left(K_{i}\right)$ for $i=0,1, \ldots, m$.

Since $\varphi_{1}$ and $\varphi_{2}$ are equally distributed it follows that for every $I \subset\{0,1$, $\ldots, m\}$

$$
v_{1}\left(\varphi_{1}^{-1}\left(\bigcup_{i \in I} K_{i}\right)\right)=v_{2}\left(\varphi_{2}^{-1}\left(\bigcup_{i \in I} K_{i}\right)\right)=v_{2}\left(\bigcup_{i \in I} S_{i}\right) .
$$

The $K_{i}$ 's are disjoint, hence

$$
v_{1}\left(\varphi_{1}^{-1}\left(\bigcup_{i \in I} K_{i}\right)\right) \geqq v_{1}\left(f^{-1}\left(\bigcup_{i \in I} K_{i}\right)\right)=\sum_{i \in I} v_{1}\left(f^{-1}\left(K_{i}\right)\right)=\sum_{i \in I} \alpha_{i} .
$$


We now have

$$
\begin{aligned}
& v_{2}\left(\bigcup_{i \in I} S_{i}\right) \geqq \sum_{i \in I} \alpha_{i}, \text { for all } I \subset\{0,1, \ldots, m\}, \text { and } \\
& v_{2}\left(\bigcup_{i=0}^{m} S_{i}\right)=1=\sum_{i=0}^{m} \alpha_{i},
\end{aligned}
$$

therefore we may apply the Lemma to get a partition $\left\{T_{i}\right\}_{i=0}^{m}$ of $A_{2}$ such that $T_{i} \subset S_{i}=\varphi_{2}^{-1}\left(K_{i}\right)$ and $\nu_{2}\left(T_{i}\right)=\alpha_{i}$.

Define $\left.g\right|_{T_{i}}$ to be an integrable selection of the correspondence $\varphi_{2} \cap K_{i}$; since $\varphi_{2}$ is integrably bounded, this selection is possible [Hildenbrand (1974, D.II.4, theorem 2)]. Then $g \in \mathscr{L}_{\varphi_{2}}$ and it is easy to verify that $\rho(\mathscr{D} f, \mathscr{D} g)<\varepsilon$. Q.E.D.

\section{Proof of Theorem 2}

Let $x \in \int \varphi_{1}$ and let $f \in \mathscr{L}_{\varphi_{1}}$ be such that $\int f=x$; by Theorem 1 there is a sequence $\left\{g_{n}\right\}$ converging in distribution to $f, g_{n} \in \mathscr{L}_{\varphi_{2}}$. Since $\varphi_{2}$ is integrably bounded, $\int g_{n} \rightarrow \int f=x$ [Billingsley (1968, theorem 5.4)]. Therefore $x$ is in the closure of $\int \varphi_{2}$. But $\int \varphi_{2}$ is compact [Aumann (1965, theorem 4)], and the proof is completed. Q.E.D.

\section{Proof of Theorem 3}

Let $C=\int \varphi_{1}=\int \varphi_{2}$ (by Theorem 2). $C$ is convex and compact [Aumann (1965, theorems 1 and 4)].

We proceed by induction on the dimension of $C$ in $\mathbf{R}^{t}$. If $\operatorname{dim}(C)=0$, then the theorem follows at once from Theorem 1.

Next, suppose $\operatorname{dim}(C)=n$.

Let $x \in C$ and let $f \in \mathscr{L}_{\varphi_{1}}$ be such that $x=\int f$. We must find a $g \in \mathscr{L}_{\varphi_{2}}$ such that $x=\int g$ and $\rho(\mathscr{D} f, \mathscr{D} g)<\varepsilon$.

Case $(i) . \quad x \in$ rel-int $C$. Let $r>0$ be the distance of $x$ from the boundary of $C$. Applying Theorem 1 and the integrable boundedness of $\varphi_{2}$, we can find $g^{\prime} \in \mathscr{L}_{\varphi_{2}}$ such that $\rho\left(\mathscr{D} f, \mathscr{D} g^{\prime}\right)<\varepsilon / 2$ and

$$
\left|x-\int g^{\prime}\right|<r \cdot \varepsilon / 2 \text {. }
$$

Let $y$ be the intersection of the boundary of $C$ with the half line from $\int g^{\prime}$ to $x$, and let $g^{\prime \prime} \subset \mathscr{L}_{\varphi_{2}}$ be such that $\int g^{\prime \prime}=y$. Clearly, $x=\alpha y+(1-\alpha) \int g^{\prime}$, where $\alpha<\varepsilon / 2$. Applying Lyapunov's (1940) theorem, we obtain a set $S \subset A_{2}$ satisfying $v_{2}(S)=\alpha, \int_{S} g^{\prime}=\alpha \int g^{\prime}$ and $\int_{S} g^{\prime \prime}=\alpha \int g^{\prime \prime}$. Define $g$ by

$$
g(a)= \begin{cases}g^{\prime}(a), & a \notin S, \\ g^{\prime \prime}(a), & a \in S,\end{cases}
$$

then clearly $\int g=x$ and $\rho(\mathscr{D} f, \mathscr{D} g)<\varepsilon$.

Case (ii). $x \notin$ rel-int $C$. Let $q$ define a supporting hyperplane such that $q \cdot x=\max q \cdot C$. 


\section{Denote}

$$
\hat{\varphi}_{i}(a)=\left\{y \in \varphi_{i}(a) \mid q \cdot y=\max q \cdot \varphi_{i}(a)\right\}, \quad \text { for } \quad a \in A_{i}, \quad i=1,2 .
$$

By Hildenbrand (1974, D.II.3, proposition 3), $\hat{\varphi}_{i}$ have measurable graphs. Also, $\hat{\varphi}_{i}$ are integrably bounded and closed-valued, and $f \in \mathscr{L}_{\hat{\varphi}_{1}}$ [by Hildenbrand (1974, D.II.4, proposition 6)].

We now claim that $\hat{\varphi}_{1}$ and $\hat{\varphi}_{2}$ are equally distributed. To see this, apply ${ }^{3}$ Theorem 4 to get decompositions $\varphi_{i}=\psi \circ h_{i}$, then $\hat{\varphi}_{i}=\hat{\psi} \circ h_{i}$, where

$$
\hat{\psi}(t)=\{y \in \psi(t) \mid q \cdot y=\max q \cdot \psi(t)\} .
$$

But $\operatorname{dim}\left(\int \hat{\varphi}_{1}\right)<n$, hence by the induction hypothesis there is a $g \in \mathscr{L}_{\hat{\phi}_{2}} \subset$ $\mathscr{L}_{\varphi_{2}}$ such that $x=\int g$ and $\rho(\mathscr{D} f, \mathscr{D} g)<\varepsilon$. Q.E.D.

Before proving Theorem 4, we have to introduce the concepts of topology and Borel $\sigma$-field on $\mathscr{C}^{l}$, the family of closed subsets of $\mathbf{R}^{l}$. The usual way to do this is the following [for discussion, proofs and references see Hildenbrand (1974, B.II)].

Let $X=\mathbf{R}^{l} \cup\{\infty\}$ be the one-point compactification of $\mathbf{R}^{l}$, then $X$ is a metric space (it is homeomorphic to the unit sphere of $\mathbf{R}^{l+1}$ ). Let $\mathscr{K}$ be the family of its closed (i.e., compact) non-empty subsets, endowed with the Hausdorff metric (or, equivalently, with the topology of 'closed convergence'). Let $\mathscr{B}(\mathscr{K})$ be its Borel $\sigma$-field, i.e., generated by the open subsets of $\mathscr{K}$. It follows from a result of Dubins and Orenstein [Debreu (1967, (3.1))] that $\mathscr{B}(\mathscr{K})$ is generated by the sets $V^{w}$ for all open subsets $V$ of $X$, where

$$
V^{w}=\{K \in \mathscr{K} \mid K \cap V \neq \phi\} .
$$

To each closed subset $C$ of $R^{l}$ (i.e., $C \in \mathscr{C}^{l}$ ), we associate the member $C \cup\{\infty\}$ of $\mathscr{K}$; then $\mathscr{C}^{l}$ can be regarded as a subset of $\mathscr{K}$, namely

$$
\{K \in \mathscr{K} \mid \infty \in K\} \text {. }
$$

The topology of 'closed convergence' on $\mathscr{C}^{l}$ is then the induced topology from $\mathscr{K}$, and $\mathscr{C}^{l}$ is a compact metric space. Moreover, let $\mathscr{B}\left(\mathscr{C}^{l}\right)$ be its Borel $\sigma$-field, then it is easy to see that

$\mathscr{B}\left(\mathscr{C}^{l}\right)$ is generated by the sets $U^{w}$ for all open subsets $U$ of $R^{l}$, where $U^{w}=\left\{C \in \mathscr{C}^{l} \mid C \cap U \neq \phi\right\}$.

For any closed-valued correspondence $\varphi:(A, \mathscr{A}, v) \rightarrow \mathbf{R}^{l}$, let $h_{\varphi}:(A, \mathscr{A}, v) \rightarrow$ $\mathscr{C}^{l}$ denote the corresponding point-valued function, i.e., the function defined by $h_{\varphi}(a)=\varphi(a)$ for all $a \in A$.

\section{Proof of Theorem 4}

The 'if' part is immediate.

To prove the converse, let $h_{i}=h_{\varphi_{i}}$ be defined as above, and let $\psi:\left(\mathscr{C}^{l}\right.$, $\left.\mathscr{B}\left(\mathscr{C}^{l}\right)\right) \rightarrow \mathbf{R}^{l}$ be defined by $\psi(C)=C$ for all $C \in \mathscr{C}^{l}$; obviously $\varphi_{i}=\psi \circ h_{i}$.

${ }^{3}$ The proof of Theorem 4 does not depend on Theorem 3 . 
Let $U$ be an open subset of $\mathbf{R}^{l}$, then $\psi^{-1}(U)=U^{w} \in \mathscr{B}\left(\mathscr{C}^{l}\right)$. Since $\mathscr{C}^{l}$ is a complete separable metric space and $\psi$ is a closed-valued correspondence, it follows from [Hildenbrand (1974, D.II.3, proposition 4(b)] that $\psi$ has a measurable graph.

Again, let $U$ be an open subset of $\mathbf{R}^{l}$, then $h_{i}^{-1}\left(U^{w}\right)=\varphi_{i}^{-1}(U)$. Since $\varphi_{i}$ has measurable graph, $\varphi_{i}^{-1}(U) \in \mathscr{A}$ by Hildenbrand (1974, D.II.3, proposition 4(a)), hence $h_{i}$ is a measurable function.

Moreover, $\mathscr{D} h_{i}\left(U^{w}\right)=\mathscr{D} \varphi_{i}(U)$, hence $\mathscr{D} \varphi_{1}=\mathscr{D} \varphi_{2}$ implies $\mathscr{D} h_{1}=\mathscr{D} h_{2}$ by $(*)$ Q.F.D.

The Corollary now follows immediately from Theorem 4 and its proof.

\section{Acknowledgement}

The authors wish to express their deep gratitude to Professor W. Hildenbrand for introducing them to these problems and for many enlightning conversations. We are also indebted to Professor R.J. Aumann for a simplification in the proof of Theorem 4.

\section{References}

Aumann, R.J., 1965, Integrals of set-valued functions, J. Math. Anal. and Appl. 12, 1-12.

Billingsley, P., 1968, Convergences of probability measures (Wiley, New York).

Debreu, G., 1967, Integration of correspondences, Proc. 5th Berkeley Symp. II, 1, 351-372.

Halmos, P.R. and H.E. Vaughan, 1950, The marriage problem, Am. J. of Math. 72, 214-215.

Hart, S., W. Hildenbrand and E. Kohlberg, 1974, On equilibrium allocations as distributions on the commodity space, Journal of Mathematical Economics 1, preceding article.

Hildenbrand, W., 1974, Core and equilibria in a large economy (Princeton University Press, Princeton).

Kannai, Y., 1970, Continuity properties of the core of a market, Econometrica 38, 791-815.

Lyapunov, A., 1940, Sur les fonctions-vecteurs completement additives, Bull. Acad. Sci. USSR, Ser. Math. 4, 465-478. 